\title{
MODELING OPERATION OF LIQUID METAL FUSES WHEN BREAKING OVERCURRENTS
}

\author{
A $V$ Kuznetsov $^{1}, D S$ Aleksandrov $^{1}$ and $Y P$ Yurenkov $^{1, *}$ \\ ${ }^{1}$ Ulyanovsk State Technical University
}

\begin{abstract}
This paper shows that successful switching of extremely high short-circuit currents I> $50 \mathrm{kA}$ can be achieved by joint operation of a liquid-metal self-resetting current limiter and a circuit breaker connected in series. The type NFU-225 device from Mitsubishi was taken as an example. The time-current characteristic of joint operation of a liquid-metal self-resetting current-limiting device and a circuit breaker was compiled. However, further in the article physical processes occurring in a liquid-metal self-resetting current limiter with a complete transformation of fusible unit are considered. The result of work is modelling of operation of liquid-metal fuses when overcurrents are switched off based on the pilot studies obtained by the Japanese scientists. It is proposed to simulate the break process not at every time moment, but at specific time moments (reference points). At other time moments, current and voltage should be considered as approximately linearly changing characteristics. The work of current limiter can be represented by three stages: the pre-arc, the main arc and the final arc. If the current density is less than $1000 \mathrm{~A} / \mathrm{mm} 2$, then the pre-arc operation stage of the current limiter includes the following sections for heating the fusible unit: primary heating to the melting temperature; melting and its transition to liquid state; secondary heating to evaporation temperature; evaporation of fusible unit.
\end{abstract}

\section{Introduction}

Implementation of new electrical equipment and promising circuit solutions, for example, by means of:

- Increasing the unit capacity of power transformers to $\mathrm{Sn}=4 \mathrm{MVA}$;

- Switching of two or three power transformers to parallel operation;

- Using powerful autonomous energy systems (ship power systems);

- Advancement of powerful converters that provide conversion of alternating current to direct current,

depends on the presence of high current and currentlimiting electrical devices which enable switching high short circuit (SC) currents.

Successful switching of extremely high short-circuit currents $I>50 \mathrm{kA}$ can be achieved by joint operation of liquid-metal self-resetting current limiter (hereinafter referred to as current limiter) and a circuit breaker connected in series. A current limiter can be a structural part of a single electrical device, and in some cases it can be used separately, operating not with one circuit breaker, but with a group of linear circuit breakers. The combination current limiter - circuit breaker opens up the possibility of using conventional circuit breakers with a relatively low switching capacity. Limitation of shortcircuit current is provided by a current limiter, and the circuit breaker completes the shutdown process, finally opening the circuit using its contact-arc damping system. Thus, the current limiter is actually an accompanying electrical apparatus to the main reusable device, the purpose of which is similar to that of a link of aM-type fuse.

The nature of joint operation of current limiter and circuit breaker is most easily understood using the combined time-current characteristics, obtained separately for the current limiter $t=f(i)$ and the circuit breaker (Fig. 1). As an example, consider an apparatus of the NFU - 225 type [1].

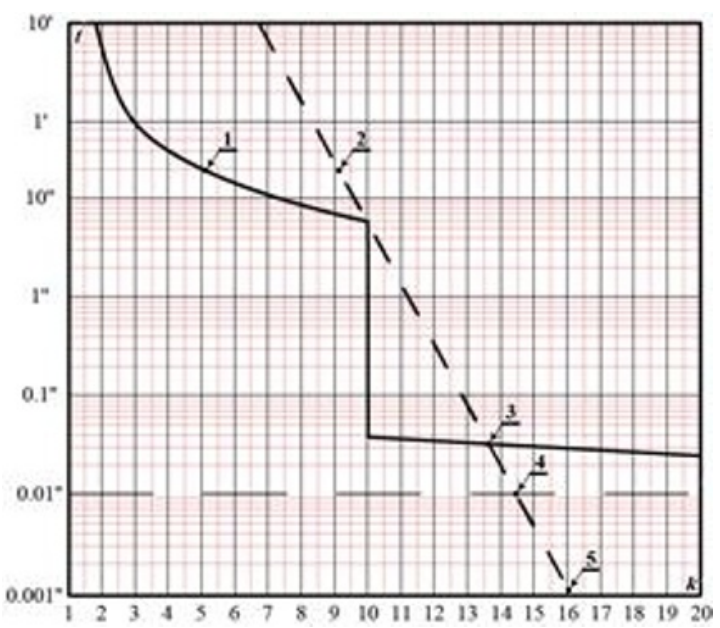

Fig. 1. Time-current characteristics of the circuit breaker (1) and current limiter (2): $t$ is the response time; $k$ is the relative short-circuit current by reference to the nominal current $I_{\mathrm{n}}$ (short circuit current multiplicity).

\footnotetext{
* Corresponding author: alxim2@mail.ru
} 
In section 2-3, where the short circuit current multiplicity $k$ is less than $15 I_{\mathrm{n}}$, the short circuit current is disconnected by a circuit breaker, and the current limiter can't operate fast enough. In section 3-5, where the short circuit current multiplicity $k>15 I_{\mathrm{n}}$, the short circuit current is breaking by the current limiter, and the circuit breaker "picks up" this process and completes it. The section 3-5 is divided into two sub-sections in terms of features of breaking the short circuit current. On the first subsection 3-4, the response time enables to reignite the arc at the location of fusible unit of the current limiter, since the possibility of "picking up" the short circuit current by a circuit breaker is limited by its relatively long operation. On the second subsection 4-5, the response time of current limiter is less than $10 \mathrm{~ms}$, and the current passed through it is quite sufficient for reliable operation of the circuit breaker and completion of breaking after the first passing of current through zero.

The relevance of studying arc phenomena in current limiters is associated not only with the need to create them, but also with economic issues. In view of this, it becomes imperative to reduce the amount of expensive development work and testing due to wider use of analytical and numerical calculations.

The result of arc phenomena study should be a mathematical model that allows one to solve practical problems that arise not only for designers, but also for testers of electrical devices, designers of electrical systems. Creation of a mathematical model of arc phenomena in liquid-metal current-limiting devices is extremely complicated and the repeated attempts to obtain it [2-6] were unsuccessful.

Next, physical processes occurring in a current limiter with a complete conversion of a fusible unit of diameter $d$ are considered under the following triggering conditions:

- Single-phase short-circuit currents in alternating current circuit;

- Limiting short-circuit currents with multiplicity $k$ more than 100 ;

- Operation of circuit breaker is not taken into account.

Physical processes were studied with various degrees of depth in [2,7-10]. Therefore, there arises a task for comprehensive qualitative character of representation and generalization of physical phenomena in the current limiter at the main stages of work by summarizing the experimental research data presented in [1,4,11-16].

The analysis of the listed information sources shows that the work of current limiter can be represented in three stages: pre-arc, main arc and final arc.

\section{Materials and Methods}

\section{Pre-arc operation stage.}

Its time duration depends on the density of shortcircuit current flowing through the lateral and uniform cross-section of the fusible unit with diameter $d$ and length $l_{\mathrm{fe}}$ (Fig 2). An approximate boundary of overcurrent density in the fusible unit cross section is a value equal to $j=1000 \mathrm{~A} / \mathrm{mm}^{2}$ [17].

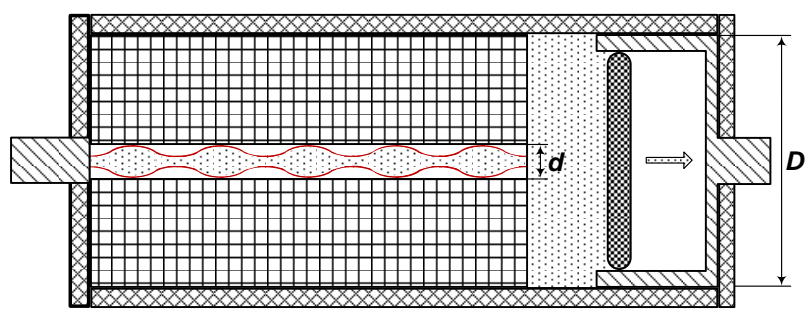

Fig. 2. The deforming impact of pinch effect on a fusible unit of diameter $d$ and length $l_{\mathrm{fe}}: D$ is the diameter of chamber for pressure transfer from liquid metal fusible unit to the buffer chamber.

Consider a short-circuit current range with a current density less than $1000 \mathrm{~A} / \mathrm{mm}^{2}$.

If the current density is less than $1000 \mathrm{~A} / \mathrm{mm}^{2}$ [17], then the pre-arc stage of current limiter operation includes the following heating sections of the fusible unit (Fig.3):

- Primary heating to a melting point $\mathbf{1}$;

- Melting and its transition to liquid state 2;

- Secondary heating to evaporation temperature 3;

- Evaporation of fusible unit 4.
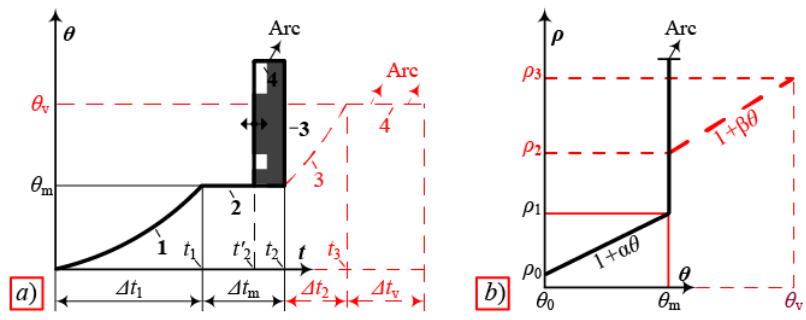

Fig. 3. Relationship between temperature of fusible unit $\theta=f(t)$ (a) specific resistance of metal and the heating temperature (b) $\rho=f(\theta)$.

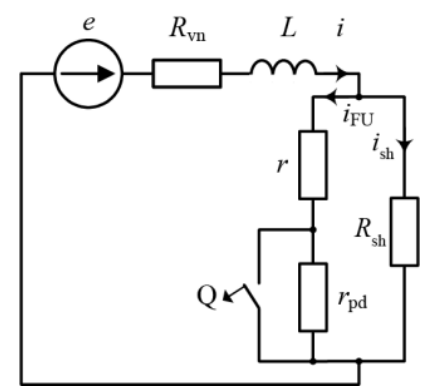

Fig. 4. Equivalent circuit for the main arc stage.

The primary heating section of fusible unit of current limiter 1 of $\Delta t_{1}$ duration, in which temperature varies from the initial temperature $\theta_{\text {in }}$ to the melting temperature $\theta_{\mathrm{m}}$.

The model of a homogeneous body [17] is adopted as a mathematical model of thermal process of the fusible unit. Heating of fusible unit is adiabatic, since the circuit break by a current limiter begins from current exceeding the maximum operating current of the electromagnetic breaker equal to $I_{\mathrm{av}} \geq 10-12 I_{\mathrm{n}}$. In addition, due to the small temperature range of the primary heating section 
$\theta_{\mathrm{m}}-\theta_{0}$, the change in the fusible unit resistance $r$ can be considered linear in the first approximation:

$$
r_{\theta}=r_{\theta 0}(1+\alpha \tau)
$$

where $r_{\theta}$ is resistance at the current fuse temperature;

$r_{\theta 0}$ is resistance at ambient temperature $\theta_{\mathrm{amb}}$;

$\alpha$ is the temperature coefficient of resistance of unmelted metal of fusible unit;

$\tau=\theta-\theta_{\mathrm{amb}}$ is the difference between the current temperature over the ambient temperature, and the heat capacity $C$ is constant and equal to heat capacity at the initial temperature of the fusible unit $\theta_{0}$.

The melting section of the fusible unit 2 (Fig. 3, a) of duration $\Delta \mathrm{t}_{\mathrm{m}}$, at which temperature remains constant and equal to the melting temperature of metal $\theta_{\mathrm{m}}=$ const.

After reaching the melting temperature $\theta_{\mathrm{m}}$, the fusible unit begins passing to a liquid state. At the melting temperature, the specific resistance of the molten metal will be much larger than the specific resistance of a solid metal. Consequently, the resistance of fusible unit consists of resistances of its two parts: the inner part, consisting of liquid metal with cross section $\mathrm{s}_{2}$ and specific resistance $\rho_{2}$; and the outer part, which consists of a still hard metal with cross section $s_{1}$ and specific resistance $\rho_{1}$. The resistance behaviour during melting of fusible unit is shown in Fig. 3, b.

The melting time $\Delta t_{\mathrm{m}}$ can be determined by integrating a differential equation compiled based on the energy balance: the heat of melting and the energy released by the short-circuit current, without taking part of it into the associated elements of the current limiter.

The section of secondary heating of the fusible unit $\mathbf{3}$ (see Fig. 3) of duration $\Delta t_{2}$, in which the temperature varies from the melting temperature $\theta_{\mathrm{m}}$ to the evaporation temperature of liquid metal $\theta_{\mathrm{v}}$.

For a short time $\Delta t_{2}$, the molten metal of the fusible unit continues to be heated by current. The specific resistance again increases linearly from $\rho_{1}$ to $\rho_{2}$ (see Fig. $3, \mathrm{~b})$. As in the 1 st heating section the model of a homogeneous body [17] is adopted as a mathematical model of thermal process of the fusible unit. Heating of fusible unit is also adiabatic. The change in resistance of fusible unit can be taken linear

$$
r_{\theta}=r_{\theta m}\left[1+\beta\left(\theta-\theta_{m}\right)\right]
$$

where $r_{\theta}$ is the resistance at the current fuse temperature;

$r_{\theta \mathrm{m}}$ is the resistance at temperature of the melted element $\theta_{\mathrm{m}}$;

$\beta$ is the temperature coefficient of resistance of a fusible unit in a liquid state;

$\theta$ is the current temperature.

The heat capacity is assumed to be constant and equal to the average heat capacity $C=\left(C_{\theta \mathrm{m}}+C_{\theta \mathrm{v}}\right) / 2$ at the melting and boiling points of liquid metal.

The list of conditions for obtaining the instantaneous short-circuit current $i_{\mathrm{t} 3}$ at time $t_{3}$ in general remains the same as that considered during analysis the processes of the primary heating section $\mathbf{1}$ and melting section $\mathbf{2}$. The short-circuit current is set by the external inductive resistance of the circuit $X_{\mathrm{ext}}$ and does not depend on the resistance of the fuse element. If necessary, the resistance of the fusible unit $r$ can be taken as the average value $r=\left(r_{2}+r_{4}\right) / 2=$ const during the secondary heating time $\Delta t_{2}$ to the boiling temperature $\theta \mathrm{v}$.

If the relative short-circuit current is not less than fifty times greater than the nominal current of the current limiter $I_{\mathrm{n}}$, then the instantaneous SC current $i_{\mathrm{t} 3}$ calculated by this method and the time of its occurrence $t_{3}$ will be overestimated due to appearance of magnetohydrodynamic instability of liquid metal already in the second section 2 of $\Delta t_{\mathrm{m}}$ duration (see Fig. 5, a). The current limiter in this case operates not as a result of evaporation of liquid metal, but actually due to destruction of integrity of fusible unit.

Upon occurrence of overcurrent with a density more than $1000 \mathrm{~A} / \mathrm{mm}^{2}$, the fusible unit of diameter $d$ and length $l_{\mathrm{fe}}$ is heated to complete dilution. As a result of pinch effect (self-compression), it presents a chain of narrow straits and metal droplets connected in series and bounded by walls of channel with diameter $d$ of the current limiter (Fig. 2). The longitudinal (tensile) component of force that arises in this case acts on the molten liquefied conductor in such a way that its wider part (drop) expands further, and the narrow part narrows further. This accelerates the rupture of circuit as a result of accelerated heating of straits and due to appearance of a breaking electrodynamic force in the places of narrowing the streamlines.

Figure 2 shows five drops and straits in order to clearly demonstrate the impact of pinch effect on a fusible unit in a liquid state. In fact, the size of one drop and strait adjacent to it does not exceed $0.5 \mathrm{~mm}$ [8]. Therefore, at least forty drops and straits may appear along the length of the fusible unit, for example, with a length $l_{\mathrm{fe}}=2 \mathrm{~cm}$.

So, if the current density is more than $1000 \mathrm{~A} / \mathrm{mm}^{2}$ [17], then due to the pinch effect, the pre-arc stage of the current limiter includes a smaller number of heating sections of fusible unit (Fig. 3, a):

- Primary heating to melting temperature 1;

- Melting and its transition to a liquid state 2.

Duration of stage $\mathbf{2}$ corresponds to the time range $\left(t_{2}-t_{1}\right) \div\left(t_{2}^{\prime}-t_{1}\right)$ (see Fig. 3, a), since due to electrodynamic forces and accelerated heating of straits, short arcs can occur before the maximum time of the melting section $t_{2}$ and transition of fusible unit into a liquid state.

It is a very difficult task to take into account the impact of pinch effect on decreasing the instantaneous value of SC current $i_{\mathrm{t} 3}$ and the time of its occurrence $t_{3}$. The simplest solution can be obtained by introducing the correction coefficient $k_{\mathrm{t} 3}^{\prime}$ or $k_{\mathrm{t} 3}$, taking into account the effect of self-compression of liquid metal of the fusible unit. The value of correction coefficient can be estimated from experimental data on the actually transmitted current $i_{\mathrm{t} 3}$. The actual values of arc appearance time $t_{2}^{\prime}$ or $t_{2}$ are found by multiplying the calculated time of evaporation start $t_{3}$ by the correction coefficient $k_{\mathrm{t} 3}^{\prime}$ or $k_{\mathrm{t} 3}$, i.e., one should perform the following steps: 


$$
\left\{\begin{array}{c}
t_{2}^{\prime}=k_{\mathrm{t}_{3}}^{\prime} t_{3} \\
o r \\
t_{2}=k_{\mathrm{t}_{3}} t_{3}
\end{array} .\right.
$$

Basing on consideration of thermal processes and assessment of their influence on electromagnetic processes, we can conclude that it is possible, as the first approximation, to construct a mathematical model based on two reference points with coordinates:

- First point - the process start $t_{0}=0 \rightarrow\left\{\begin{array}{l}i=0 \\ u=0\end{array}\right.$.

- Second point - instantaneous voltage at time $t_{2}^{\prime}$ or $t_{2}$ preceding arcing (the emergence of a chain of short arcs)

$$
t_{2}^{\prime} \div t_{2} \text { ort } t_{3} \rightarrow\left\{\begin{array}{c}
i=i_{\mathrm{tr}} \\
u=i_{\mathrm{tr}_{\mathrm{t}_{2}}} r_{t r} i_{\mathrm{t}_{2}} \text { or } i_{\mathrm{tr}} r_{\mathrm{t}_{3}}
\end{array}\right.
$$

The following notations are used in the formulas:

- $i$ and $u$ are the instantaneous SC current or the instantaneous current at the terminals of current limiter at the initial moment or at the time immediately preceding the beginning of arcing;

- $i_{\mathrm{tr}}$ is the transmitted current;

- $r_{\mathrm{t}^{\prime} 2}, r_{\mathrm{t} 2}, r_{\mathrm{t} 3}$ are the resistances of fusible unit at the corresponding times $t_{2}^{\prime}, t_{2}, t_{3}$.

The presented discussion shows a prospect for constructing a simple mathematical model of the prearcing stage using two reference points by approximating the short-circuit current $i$ and voltage $u$ at the terminals of the current limiter by a straight line at a time interval of $0-\left(t^{\prime} 2 \div t_{2}\right)$ or time $0-t_{3}$ depending on the presence or lack of pinch effect.

The necessary refinement of mathematical model is performed by transition from discrete to continuous representation of current and voltage by functional dependencies:

- $i=f(t)$, obtained for electrical short-circuited circuit without taking into account the resistance of fusible unit $r$;

- $u=f(t)$ voltages on current limiter terminals.

The main arc stage of the current limiter

Its time duration depends on the speed of switching the main part of external circuit current $i$ to the branch of the shunt resistor $R_{\text {sh }}$.

Consider the range of SC currents with current density more than $1000 \mathrm{~A} / \mathrm{mm}^{2}$.

At the locations of narrow straits connected by drops, short arcs appear not simultaneously, but one after another over a very short period of time. Addition of every new arc increases the voltage across the arc gap in steps by a value not less than a value of the nearelectrode voltage drop $U \mathrm{e}=20-25 \mathrm{~V}$ in each of them. As a result, before formation of an arc along the entire length of a fusible unit with a diameter $d$ of length $l_{\mathrm{fe}}$ (see Fig 2), a large number of short arcs are formed in places of straits connected by drops with a total voltage of $U_{\Sigma}=\sum_{1}^{\mathrm{n}} U_{\mathrm{i}}$, where $U_{\mathrm{i}} \approx 20-25 \mathrm{~V}$ is voltage of one short arc. Due to the small size of narrow straits and drops of liquid metal, not exceeding fractions of a millimeter, and a sufficiently extended liquid metal element with a diameter $d$ and length $l_{\mathrm{fe}} \geq 1-2 \mathrm{~cm}$, an overvoltage of an order of one to two kilovolts $U_{\Sigma} \approx 1-2$ $\mathrm{kV}$ appears. The time $t_{\mathrm{U} \Sigma}$, when the overvoltage $U_{\Sigma}$ appears, is negligibly little different from the time $t=t_{3}$ or $t=\left(t_{2}^{\prime} \div t_{2}\right)$ (see Fig. 3, a) due to the high growth rate of voltage on the contact gap of the current limiter, determined by the rate of introduction of elementary arcs. This implies the validity of the following expression

$$
t_{U_{\Sigma}} \approx\left\{\begin{array}{c}
\left(t_{2}^{\prime} \div t_{2}\right) \\
o r \\
t_{3}
\end{array} .\right.
$$

The duration of increase of the total arc voltage in the range $0<u_{\mathrm{d}}<U_{\Sigma}$ does not exceed tens of microseconds. It can be estimated by the expression [1] $t=u_{\mathrm{d}} /\left(U_{\mathrm{m} \varepsilon} \varepsilon\right)$, where $U_{\mathrm{m}}$ is the amplitude of phase voltage of network, and $\varepsilon>1000 \mathrm{~s}^{-1}$ is the relative rate of increase of voltage across the arc. Therefore, limiting the overvoltages in current limiters with a complete conversion of fusible unit with diameter $d$ and length $l_{\mathrm{fe}}$ provides a presence of a shunt resistor $R_{\text {sh }}$ (Fig. 4). Acceptable overvoltage values can be obtained if $R_{\text {sh }}$ of the shunt resistor is chosen so that [15]

$$
U_{p h} / I_{R s h}<R_{s h}<3 U_{p h} / I_{R s h},
$$

where $U_{\mathrm{ph}}$ is the acting value of the phase voltage of network;

$I_{\text {Rsh }}$ is the acting value of the shunt current, not exceeding the current of maximum switching capacity of circuit breaker and the transmitted current $i_{\mathrm{t} 3}$.

The main arc stage of the current limiter is completed at time $t_{4}$, when the resistance of current limiter is actually equal to the resistance of the shunt resistor $R_{\mathrm{sh}}$. The time $t_{4}$ can be determined by knowing the behaviour of external circuit current $i$, the current of current limiter $i_{\mathrm{FU}}$, and the shunt current $i_{\mathrm{sh}}$. This behaviour can be found if the behaviour of transition process in the circuit shown in Fig. 4 is known.

High-temperature plasma during the main arc stage of the current-limiting device evaporates part of material by heating the adjacent surface of ceramic sleeve. Thus, the diameter $d$ of the fusible unit increases to a value of $d_{\mathrm{i}}$, which during subsequent shutdowns will lead to an increase in the transmitted current $i_{\mathrm{FU}}$ and, accordingly, to an increase in the released energy. Starting from some $n$-th break of short circuit current, the diameter $d_{n}$ of the fusible unit will increase so much that due to the increased transmitted current $i_{\mathrm{t} 3}$, the pressure inside the volume of the fusible unit will increase to an unacceptable value according to the strength conditions of the ceramic sleeve. This will lead to mechanical 

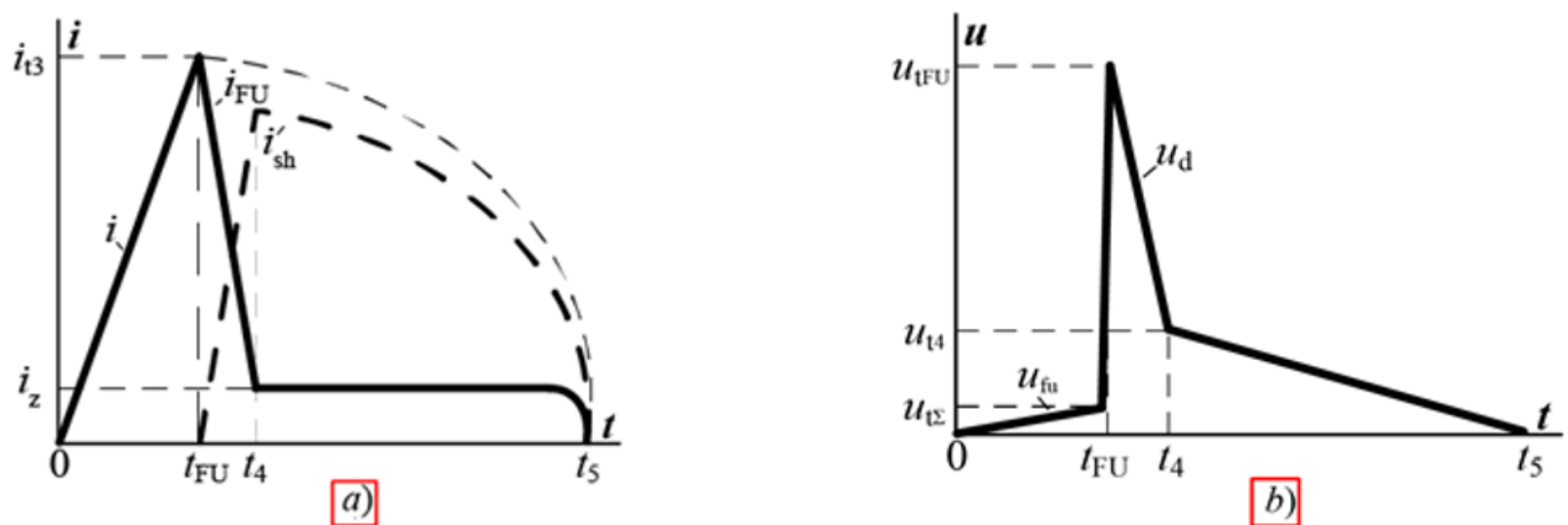

Fig. 5. Change in current of short-circuit at circuit break (a) and voltage at the terminals of current limiter (b).

destruction of the current limiter. Therefore, any liquidmetal current limiter has a specific resource in terms of the number of breaks of the limiting short-circuit currents.

\section{The final arc stage of the current limiter}

It occurs within the time interval $t_{4}-t_{5}$ (Fig. 5) and is characterized by burning of an arc in a current limiter at a significantly lower current $i_{\mathrm{z}}$ than the value of the transmitted current $i_{\mathrm{FU}}$, when the relationship $i_{\mathrm{z}} \ll i_{\mathrm{FU}}$ is fulfilled. Therefore, the shunt current $i_{\mathrm{sh}}$ is actually equal to the current of external circuit $i$, i.e., $i_{\mathrm{sh}} \approx \mathrm{i}$. Since the shutdown process is considered in an alternating current circuit, at time $t_{5}$ currents of external circuit, current limiter and shunt undergo a natural transition through zero.

\section{Conclusions}

1. A difficult problem of modeling pre-arc and arc processes in a liquid metal current limiter can be solved using a systematic approach, the initial stage of which is to qualitatively and fully consider arc phenomena.

2. The current level of knowledge does not allow constructing a generalized mathematical model of a liquid-metal current limiter suitable for calculating shortcircuit current breaking process. Therefore, the formation of model should be carried out, focusing on the simplest structure of model, taking into account the basic physical processes and combining them with sufficient depth of their description.

3 . It is proposed to simulate the shutdown process not at every time moment, but at specific time moments (reference points). At other time moments, current and voltage should be considered as approximately linearly changing characteristics.

4. The model of circuit break by a current limiter should be implemented as follows:

- Current, voltage and pressure should be considered;

- They should be determined not over the entire time period of the short-circuit current break, but only at the reference, specific time points. In the intervals between specific times, the currents and voltages are represented by straight lines;
- One should take into account the change in pressure inside the volume of the fusible unit during the main and final arc stages, caused not only by vapor of the liquid metal, but also by vapors of ceramic sleeve.

The model should be constructed, focusing, first of all, on the following specific control points of the general break process, delimiting its individual stages.

5. Further improvement of model should be carried out taking into account the nature of the influence of processes on successful operation of the current limiter in:

- Determining the limiting diameter of the fusible unit at which the current limiter can successfully turn off the limit value of the short circuit current;

- The impact of shock waves on the process of arc burning and on mechanical strength of elements of current limiter.

\section{Acknowledgements}

This work was funded by RFBR, project number 19-3890307.

\section{References}

[1] S. Dzezhbicki, E. Valchuk, Current-limiting AC switches: trans. from Polish (Leningrad: Energoizdat, Leningrad branch, 116, 1982).

[2] P.A. Kulakov, O.Ya. Novikov, V.I. Prikhodchenko, V.V. Tanaev, High-current fuses, current limiters and switches with liquid metal contacts (Moscow: Informelectro, 49, 1984).

[3] V.V. Tanaev, A.F. Kuzmin, Features of arc burning in devices with liquid metal contacts, Electrotechnical industry 6, 5-6 (1982).

[4] S.L. Kuzhekov, B.N. Vasil'ev, N.N. Kurov, Calculation of dynamic processes occurring during the operation of liquid-metal self-resetting fuses, Proc. of Higher Educ. Institutions, Electromechanics 2, 131-134 (2012).

[5] S.L. Kuzhekov, B.N. Vasil'ev, N.N. Kurov, Performance evaluation of a self-resetting liquid metal 
fuse, Proc. of Higher Educ. Institutions, Electromechanics 2, 134-136 (2012).

[6] A.V. Kuznetsov, Y.P. Yurenkov, Mathematical model of the short circuit process in the electrical network with self-recovering current-limiting device with a liquid metal, IOP Conf. Ser.: Mater. Sci. Eng. 643, ISEPC-2019 (St. Petersburg, Russia), 012083 (2019).

[7] L. Niemeyer, Evaporation dominated high current arcs in narrow channels, PAS-98, IEEE Trans 3, 950-958 (1978).

[8] J.De. Palma, J. Gelet, Observation of arcing inside a fuse under capacitor-discharge using 1 million frames per second X-ray imaging, 19th European Conf. on Power Electronics and Applications (Warsaw, Poland), 1-10 (2017).

[9] Zhuo Yang, Hailong he, Fei Yang, Yi Wu, Mingzhe Rong, Peng Zhao, Siyu Lv, Qing Wan, A Novel Topology of Liquid Metal Fault Current Limiter for MVDC Network Applications, IEEE Transactions on Power Delivery, 1 (2019).

[10] V.P. Mesheryakov, R.T. Sibatov, V.V. Samoilov, A.S. Topchiy, Calculation of the arc current and the time of its extinction in low-voltage circuit breakers, Electrical Engineering, Low voltage devices 2, 43-49 (2008).

[11] T. Itoh, T. Miyamoto, Y. Wada, Design considerations and the applications of permanent power fuse, Conference Paper NC72 103-5 IEEE Winter Meeting (1972).

[12] T. Itoh, T. Miyamoto, Y. Wada, T. Mori, H. Sasao, Design considerations on the PPF for a control center, IEEE Transactions on Power Apparatus and Systems 4, 1292 (1973).

[13] J.G. Murray, G.A. Bronner, Proposed method of rating fuses for pulsed service, IEEE Trans. Nucl. Sci. 4, 230-234 (1971).

[14] Resettable fuse: certificate of authorship, 1479972, kl. N 01 N 87/00 (1989).

[15] K.K. Namitokov, N.A. Ilina, I.G. Shklovsky, Devices to protect semiconductor devices (Moscow: Energoatomizdat, 279, 1988).

[16] H. He, et al., Study of Liquid Metal Fault Current Limiter for Medium-Voltage DC Power Systems, IEEE Trans. Compon. Packag. Manufacturing Technol. 8, 8, 1391-1400 (2018)

[17] R. Ryudenberg, ed. K.S. Demirchan, Operational modes of electric power systems and installations: trans. from German, 3rd publ. (Leningrad: Energiya, Leningrad branch, 578, 1980). 